\title{
ON THE OPTIMIZATION OF SPATIO-TEMPORAL ANALYSIS FOR SOURCE MOTION ESTIMATION
}

\author{
O. Zugmeyer and J.P. Le Cadre
}

IRISA, Campus de Beaulieu - 35042 Rennes Cedex, France

\begin{abstract}
The estimation of source motion parameters takes a considerable importance for many areas of array processing. Especially for underwater acoustic applications where a large amount of data is available. The optimization of spatio-temporal analysis is considered, then original methods for source motion analysis are derived.
\end{abstract}

\section{Introduction}

The classical array processings rely upon short time analysis. Whatever the considered processing (e.g. classical beamforming or high-resolution method), they try to optimize a spatial contrast at a given instant. The integration time is itself limited by the basic unstationarity of the received signals (source motion, propagation effects,...). The optimal integration time is then a compromise between the accuracy of the observations (e.g. intersensor correlation estimates) and the effects of source motions. This point has been previously studied, revealing that the optimal integration time decreases as the sensor number increases [1].

However, in the general context of passive array processing (e.g. underwater acoustic) a very large amount of data is available. In order to use them, the following steps must be considered :

- derivation of a simplified model of a moving source,

- inclusion of the motion model in the source one,

- spatio-temporal analysis (source motion analysis).

The last point is also the more difficult and important one. It is then possible not only to consider very large integration times (for detection of weak sources) but also to discriminate the sources by their whole respective trajectories.

The spatio-temporal data are described by using a 2D state-space model, eventually with time-varying parameters (close sources). In this case, the instantaneous values of the parameters are estimated by using Kalman filters conditioned by the hyperparameters describing the temporal variations of the local model.

As we shall see later, the estimation of source motion paramters are basically defined for multiscale analysis. This leads us to consider the following fundamental problems

- study of the statistical properties of the spatio-temporal data,

- statistical analysis of the source motion estimates,

- optimization of the spatio-temporal analysis,

- multiscale state-space analysis.

The study of the first two questions relies on statistical perturbation analysis, whereas the two last involve heavily interpolation procedures.

\section{A simplified model of source motion}

For array processing, the relevant parameter is the spatial frequency $k_{t}$. Let $k_{t}$ the spatial frequency associated with one source at time $t$, then the following model of $k_{t}$ will be considered for the sequel :

$$
k_{t}=k_{0}+t \dot{k}
$$

The model (1) is a global linear approximation. For close sources it can be replaced by a local approximation, i.e. :

$$
k_{t+1}=k_{t}+\dot{k}_{t}
$$

For the first model, the source trajectory is defined by two parameters (resp. $k_{0}$ and $\dot{k}$ ). The problem we shall now consist in the estimation of the parameters $k$ (and $k_{0}$ ) for each source.

\section{Statistical properties of the data}

Let $\left\{X_{t}\right\}$ the array snapshots, classical analysis rely on the use of a spatial contrast at a given time. Instead of this, we shall consider the whole sequence of $\left\{X_{t}\right\}$. At a given frequency (omitted), the data are represented by the spatio-temporal estimated covariances $\hat{r}(t, m)(t:$ time, $m$ : spatial index). For a linear array, these data are deduced from the empirical covariance matrix $\hat{R}_{t}$ (estimated on $N$ snapshots) by using the classical formula [2] :

$$
\hat{r}(t, m)=\frac{1}{p-m} \operatorname{tr}\left(\hat{R} \cdot Z^{m}\right) 0 \leq m \leq p-1
$$

$Z$ : shift matrix [2], $t r:$ the trace and $p:$ sensor number. Invoking some classical results, the statistical proper- 
ties of the data $r(t, m)$ are derived [2]. The following formula is a basic one :

$\mathbb{E}\left(\tilde{r}(t, m) \tilde{r}^{*}(t, n)\right)=\frac{1}{(p-m)(p-n) \cdot n} t r\left(Z^{m} R_{t} Z^{n^{t}} R_{t}\right)$

with :

$$
\tilde{r}(t, m) \triangleq \hat{r}(t, m)-\mathbb{E}(\hat{r}(t, m))
$$

$t$ : transposition, * : transposition-conjugation.

For a unique source (of signal to noise ratio $\rho$ ) the formula (4) yields directly :

$$
\mathbb{E}\left(|\tilde{r}(t, m)|^{2}\right)=\frac{1}{N} \cdot\left(\frac{1}{p-m}(1+2 \rho)+\rho^{2}\right)
$$

Thus the variance of the estimation noise of the data $\hat{r}(t, m)$ (i.e. $\left.\mathbb{E}\left(|\tilde{r}(t, m)|^{2}\right)\right)$ increase with the spatial index $m$, the dependance upon $m$ being given by (4), (5). In particular, this variance can be much more important than the deterministic contribution of a weak source. This is a major limitation.

In the two-source case, the calculation of $\mathbb{E}\left(\tilde{r}(t, m) \cdot \tilde{r}^{*}(t, n)\right)$ yields directly :

$$
\begin{aligned}
\lim _{p \rightarrow \infty} \mathbb{E}[\tilde{r}(t, m) \tilde{r}(t, n)]= & \rho_{1}^{2} \exp \left(-2 i \pi d(m-n) k_{1, t}\right) \\
& +\rho_{2}^{2} \exp \left(-2 i \pi d(m-n) k_{2},(\mathrm{\theta})\right.
\end{aligned}
$$

( $\rho_{1}$ and $\rho_{2} s / n$ ratios of the two sources, $d$ : intersensor distance, $N=1$ ).

The eq. 6 is fundamental for the rest. It proves that the estimation noise is highly spatially correlated, its correlation matrix being proportional to the strong sources CSM. It will be then possible to cancel the effects of the noise estimation by means of a spatial analysis (e.g. beamforming, see section 7).

For the sequel, the data will be constituted of the sequences $\{y(t, m)\}_{t}(y(t, m) \triangleq \hat{r}(t, m))$. for a fixed $m$, the

\begin{tabular}{|c|c|c|}
\hline$y(t, 1)$ & $y(t+1,1)$ & $y(t+N, 1)$ \\
\hline$y(t, 1)$ & $y(t+1,2)$ & $\overline{y(1 t}+N, \overline{2})$ \\
\hline$y(t, p-1)$ & $y(t+1, p-1)$ & $y(t+N, p-1)$ \\
\hline
\end{tabular}
temporal sequence $\{y(t, m)\}_{t}$ will be simply named "file $m$ ". The organization of the data is presented below (figure 1).

Figure 1

Under a gaussian assumption for the file $m$, the following Cramer-Rao bounds for the estimation of the parameters $k_{0}$ and $\dot{k}$ (denoted $\hat{k}_{0, m}$ and $\hat{\dot{k}}_{m}$ ) on the file $m$ are given by :

$$
\begin{aligned}
\operatorname{var}\left(\hat{k}_{m}\right) & \geq \frac{6 \operatorname{var}(\hat{r}(t, m))}{4(\pi d \rho)^{2} m^{2}\left(N^{2}-1\right) N} \\
\operatorname{var}\left(\hat{k}_{0, m}\right) & \geq \frac{(2 N-1) \operatorname{var}(\hat{r}(t, m))}{4(\pi d \rho)^{2} m^{2} N(N+1)}
\end{aligned}
$$

The expression (7) of $\operatorname{var}\left(\hat{\dot{k}}_{m}\right)$ is valid at low $s / n$ ratios, by using (5) it leads to the following result :

$$
\operatorname{var}\left(\dot{\hat{k}}_{m}\right)=\frac{6}{4(\pi d \rho)^{2}\left(N^{2}-1\right) \cdot N} \cdot f_{m}(\rho)
$$

with :

$$
f_{m}(\rho)=\left(\frac{1+2 \rho}{p-m}+\rho^{2}\right) \cdot \frac{1}{m^{2}}
$$

Examinating $f_{m}(\rho)$, we see that its unique minimum is attained for a value of the spatial index $m$ comprised between $2 p / 3$ and $p-1$.

\section{Spatio-temporal representations of the data $y(t, m)$}

Assuming that the model (1) of $k_{t}$ is valid, then the 2Darray of data $\{y(t, m)\}$ can be represented by the following 2D-state space model :

$$
\mid \begin{aligned}
& X(t+1, m)=F_{1}^{m} X(t, m) \\
& X(t, m+1)=F_{0} F_{1}^{t} X(t, m) \quad 1 \leq m \leq p-1 \\
& y(t, m)=h^{*} X(t, m)+w(t, m) \quad t_{0} \leq t \leq t_{e}
\end{aligned}
$$

with ( $s$ sources) :

$$
\begin{aligned}
& F_{1}=\operatorname{diag}\left(\exp \left(2 i \pi d \dot{k}_{1}\right), \cdots, \exp \left(2 i \pi d \dot{k}_{s}\right)\right) \\
& F_{0}=\operatorname{diag}\left(\exp \left(2 i \pi d k_{1}(0)\right), \cdots, \exp \left(2 i \pi k_{s}(0)\right)\right) \\
& h^{*}=(1,1, \cdots, 1)
\end{aligned}
$$

( $\operatorname{var}(w(t, m))$ given by $(4))$.

For a time varying model of $k_{t}$ (eq. 2) the file $m$ can be modelled as the output of an unstationary AR-model, let $(m$ omitted :

$$
\left\{\begin{array}{l}
X_{t+1}=F_{t} \cdot X_{t}+W_{t} \\
Y_{t}=h_{t}^{*} X_{t}+n_{t}
\end{array}\right.
$$

with :

$$
\begin{aligned}
Y_{t} \triangleq & (y(t, m)) ; h_{t}^{*}=(y(t-1, m), \cdots, y(t-q, m)) ; \\
& X_{t}=\left(a_{1}(t), \cdots, a_{q}(t)\right)^{t}
\end{aligned}
$$

In the eq. 10, the vector $X_{\mathrm{t}}$ represents the (time-varying) AR coefficients. The transition matrix $F$ of eq. 10 is deduced from a difference equation relative to the $\dot{k}_{j}$, let (e.g.) :

$$
\nabla^{2} \dot{k}_{t}=w_{t} \Leftrightarrow \dot{k}_{t+1}=2 \dot{k}_{t}-\dot{k}_{t-1}+w_{t} ; w_{t}: \mathcal{N}\left(0, \tau^{2}\right)
$$

The parameter $\tau^{2}$ is called an hyperparameter [3] and corresponds to a smoothness prior relative to the source trajectory. A state equation is deduced from (11), let : 


$$
\mathcal{X}(t+1)=\mathcal{F}(t) \mathcal{X}(t)+G(t) W(t)
$$

with :

$$
\begin{gathered}
\mathcal{F}(t)=\left(\begin{array}{cc}
I d+J_{t} J_{t-1}^{-1} & -J_{t} J_{t-1}^{-1} \\
I d & 0
\end{array}\right) ; G(t)=\left(\begin{array}{c}
J_{t} \\
0
\end{array}\right) \\
J_{t}(i, j) \triangleq\left(\begin{array}{c}
\left.\frac{\partial a_{i}(t)}{\partial k_{j}(t)}\right)_{1 \leq i, j \leq s} \\
W \text { is } \mathcal{N}(0, B) \text { with } B=\operatorname{diag}\left(\tau_{1}^{2}, \cdots, \tau_{s}^{2}\right)
\end{array}\right.
\end{gathered}
$$

The problem we shall now deal with, is to perform separate estimations of the parameters $\dot{k}$ and $k_{0}$ (or $\dot{k}_{z}$ ). The fundamental reason of these separate estimations is the crucial problem of interferences between moving sources [1]. In a first time, the monodimensional (on a unique file $y(t, m)$ ) methods for estimating $\dot{k}_{m}$ will be briefly presented. Then, the multidimensional estimation will be considered with some care.

\section{Monodimensional methods}

A now classical method for estimating $F_{1}$ (eq. 9) consists in considering the Hankel matrix $\hat{\mathcal{H}}_{m}$ :

$$
\hat{\mathcal{H}}_{m} \triangleq \operatorname{Hank}(y(t, m))_{t}
$$

then, the eq. 9 yields :

$\mathcal{H}_{m}=\theta_{m} \cdot \mathcal{X}_{m}, \theta_{m}:$ observability matrix and thus :

$$
\theta_{m}^{\dagger} \cdot F_{1}^{m}=\theta_{m}^{\downarrow} \text { with } \theta_{m}^{\perp}=Z \theta_{m}, \theta_{m}^{\dagger}=Z^{t} \theta_{m}
$$

Practically, the estimated observability matrix $\hat{\theta}_{m}$ is deduced from $\hat{\mathcal{H}}_{m}$ by means of a SVD analysis.

Another way consists in estimating an AR model directly fitted to the data $y(t, m)$. A classical performance analysis [2] leads to the following expression of $\operatorname{var}\left(\hat{\dot{k}}_{m}\right)$ :

$$
\operatorname{var}\left(\hat{\dot{k}}_{m}\right)=\sigma_{w}^{2}(m) \cdot\left\|\frac{\partial \dot{k}_{m}(\dot{h})}{\partial \dot{h}}\right\|^{2} h=\hat{h}
$$

with :

$$
\hat{h} \triangleq(y(1, m), \cdots, y(N, m))^{t}
$$

The calculation of (14) is rather tedious [2], [4] but leads to an interesting result. For low $s / n$ ratios, one has :

$$
\operatorname{var}\left(\hat{\dot{k}}_{m}\right)=\text { cst } \cdot f_{m}(\rho), f_{m}(\rho) \text { defined by }(8)
$$

leading to the same conclusions for the choice of the "optimal" spatial index $m$. This choice amounts to optimize the compromise between the dilatation factor of the analysis scale ( $\dot{k}$ becomes $m \dot{k}$ (eq. 9 ) on the file $m$ ) resulting in the "geometric" factor $1 / \mathrm{m}^{2}$ and the estimation noise power on $\{y(t, m)\}_{t}$.

However, the proposed algorithm treats separately each file $m$. It is possible to define numerous methods in order to achieve a fusion of the estimates $\hat{\dot{k}}_{m}$ [2] ; however a coherent approach seems more promissing.

\section{Multiscale estimation of the parameters $\dot{k}$}

We shall now deal with a coherent multidimensional of the $\left\{\dot{k}_{j}\right\}_{j=1}^{s}$. The proposed method relies heavily on an interpolation procedure, presented below.

By using eq. 9, the following interpolation formulas are valid for the state of the file $m$ :

$$
X\left(t+m_{0} / m, m\right)=F_{1}^{m_{0}} X(t, m) \quad m \geq m_{0}
$$

According to (9) and (15), the interpolated data $\tilde{Z}(t, m)$ (noise free), satisfied to :

$$
\begin{aligned}
\tilde{z}\left(t+\frac{m_{0}}{m} \cdot j, m\right) & =h^{*} X\left(t+\frac{m_{0}}{m} j, m\right) \\
& =h^{*} F_{1}^{j m_{0}} X(t, m)
\end{aligned}
$$

Consequently, thanks to the Cayley-Hamilton theorem, there exist coefficients $a_{j}\left(t, m_{0}\right)$ s.t. :

$$
\begin{aligned}
z(t, m)= & \sum_{j=1}^{s} a_{j}\left(t, m_{0}\right) \tilde{z}\left(t-\frac{m_{0}}{m} j, m\right) \\
& 1 \leq m \leq p-1
\end{aligned}
$$

Note that the above relation is valid whatever $m$ and with the same coefficients $\left\{a_{j}\left(t, m_{0}\right)\right\}$. Now, the interpolated data $\tilde{y}(s, m)$ on the file $m$ are defined as :

$$
\tilde{y}\left(t+\frac{m_{0}}{m} j, m\right)=\tilde{z}\left(t+\frac{m_{0}}{m} j, m\right)+\tilde{w}\left(t+\frac{m_{0}}{m} j, m\right)
$$

$\tilde{w}$ : interpolated noise.

So that finally, according to (17) and (18), the interpolated data $\tilde{y}(t, m)$ can be modelled by AR-models with a unique set of coefficients, i.e. :

$$
y(t, m)=\sum_{j=1}^{s} a_{j}\left(t, m_{0}\right) \tilde{y}\left(t-\frac{m_{0}}{m} j, m\right)+\tilde{w}(t, m)
$$

with : $m_{0} \leq m \leq m_{1}$.

The 2D-array of data (figure 1) is thus modelled by the following interpolated multidimensional system :

$$
\begin{aligned}
& \begin{array}{lll}
y(t, m)= & h_{t, m_{0}}^{*} X_{t}+w\left(t, m_{0}\right) \\
y\left(t, m_{0}+1\right)= & \tilde{h}_{t, m_{0}+1}^{*} X_{t}+\tilde{w}\left(t, m_{0}+1\right) \\
\vdots & \\
& m_{0} \leq m \leq m_{1}
\end{array} \\
& \begin{array}{l|l} 
& \begin{array}{l}
X_{t}=\left(a_{1}\left(t, m_{0}\right), \cdots, a_{s}\left(t, m_{0}\right)\right)^{t} \\
\text { with }
\end{array} \\
h_{t, m_{0}}^{*}=\left(y\left(t-1, m_{0}\right), \cdots, y\left(t-s, m_{0}\right)\right)
\end{array}
\end{aligned}
$$




$$
\mid \begin{aligned}
& \tilde{h}_{t, m_{0}+1}^{*}=\left(\tilde{y}\left(t-\frac{m_{0}}{m_{0}+1}, m_{0}\right), \cdots, \tilde{y}\left(t-\frac{m_{0}}{m_{0}+1} s, m_{0}\right)\right) \\
& \vdots
\end{aligned}
$$

The interpolated data $\tilde{Y}(t, m)$ defined by the previous eqs may be represented by the following figure (for the case of an $\mathrm{AR}(2))$.

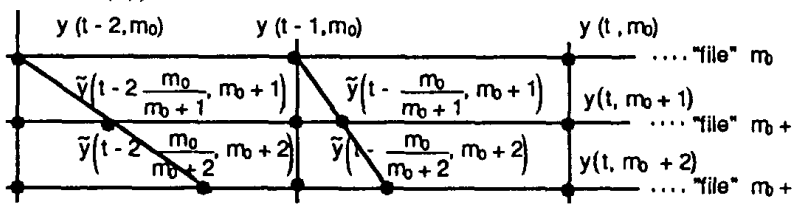

Figure 2

On the figure 2 , the initial data $y(t, m)$ are represented by the intersection of horizontal and vertical lines, the interpolated ones by the symbol $\tilde{Y}$.

The factor $m_{0} / m_{1}$ represents the maximum value of the interpolation factor. It is worth to choose a convenient value of $m_{0} / m_{1}$ by using the performance analysis of section 3. The interpolation procedure of figure 2 is reproduced at $t+1$ (or $t+D: D$ decimation factor). This scheme can be extended to the time varying modelling [4] of the eqs. 10,11,12. by using a multidimensional Kalman filter. Otherwise, the vector $X_{t}$ is estimated either by a generalized least square method or by SVD. The interpolation of data is achieved by means of classical interpolation methods (spline, etc.) since a large amount of data is available on each file $m$.

\section{On the use of the array gain}

The main limitation of the previous approach comes from the estimation noise. Fortunately, this noise has a particular and useful structure (eq. 6).

We are now trying to combine the advantages of the previous multiscale analysis with the array gain in order to cancel the estimation noise.

Consider now a linear combination of the $\tilde{Y}(t, m)$, as defined by (19) and (20), then the following equation is deduced from $(20)$ :

$$
\begin{aligned}
& D_{k_{0}}^{*} Y_{t}=\sum_{j=1}^{s} a_{j}(t) D_{k_{0}}^{*} \tilde{Y}_{t-j}+W_{t} \\
& \text { with } \\
& Y_{t} \triangleq\left(y\left(t, m_{0}\right), y\left(t, m_{0}+1\right), \cdots, y(t, m)\right)^{t} \\
& \vdots \\
& \tilde{Y}_{t-j} \triangleq\left(y\left(t-j, m_{0}\right), \tilde{y}\left(t-j m_{0} / m_{0}+1, m_{0}+1\right), \cdots,\right)^{t} \\
& \vdots \\
& D_{\dot{k}_{0}} \triangleq\left(\exp \left(-2 i x d m_{0} k_{0}\right), \cdots, \exp \left(-2 i \pi d m k_{0}\right)\right)^{t}
\end{aligned}
$$

The steering vector $D_{k_{0}}$ defined in (21) is associated with a fixed spatial frequency $k_{0}$. The noise $W_{t}$ is deduced from the interpolated noises $\tilde{w}(t, m)$ as :

$$
W_{t}=D_{k_{0}}^{*} W_{t}
$$

with :

$$
W_{t}=\left(w\left(t, m_{0}\right), \tilde{w}\left(t, m_{0}+1\right), \cdots, \tilde{w}(t, m)\right)^{t}
$$

Thanks to the noise covariance structure (eq. 6), the contribution of the strong sources, which are not in the beam of reference (i.e. $k_{0}$ ) can be greatly reduced.

In order to detect-track a weak moving source, it is worth to consider a set of beams $\left(k_{0}, k_{1}, \cdots, k_{l}\right)$ whole spatial frequencies $k_{1}$ are choosen ( $3 \mathrm{~dB}$ covering) in order to isolate a spatial sector as described below :

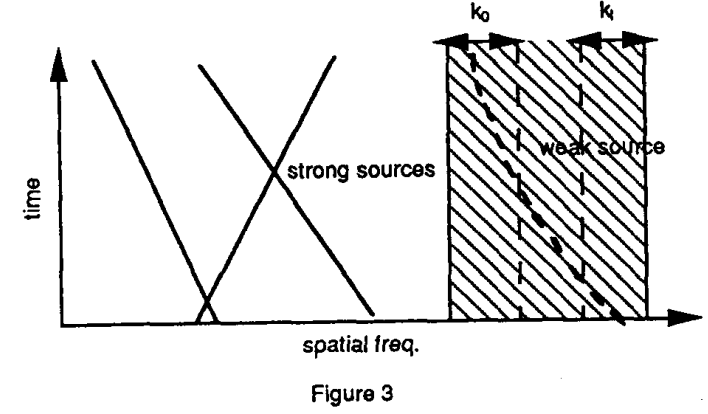

Then, the beamoutputs can be modelled by a unique (and common) AR model, let :

$$
\begin{aligned}
& D_{k_{0}}^{*} Y_{t}=\sum_{j=1}^{s} a_{j}(t) D_{k_{0}}^{*} \tilde{Y}_{t-j}+W_{0, t} \\
& \vdots \\
& D_{k_{l}}^{*} Y_{t}=\sum_{j=1}^{s} a_{j}(t) D_{k_{l}}^{*} \tilde{Y}_{t-j}+W_{l, t}
\end{aligned}
$$$$
\text { : }
$$

Obviously, the choice of the spatial frequencies $k_{0}, k_{1}, \cdots, k_{l}$ is of a fundamental importance. This part is under current investigation. This formalism may also be extended to time varying analysis.

Due to the lack of space, the simulation results are not included here however they seem quite promising [4].

Remarking that $\dot{k}$ is equal to $(\cos \theta / \lambda)$, one can derive a double interpolation scheme in order to perform a multifrequency analysis.

The aim of this strudy is not to add an extraneous method to the long list of array processing methods but to consider the general problem of spatio-temporal analysis for source motion analysis. In that meaning, it may constitute a bridge between "classical" array processing and target motion analysis.

\section{References}

[1] O. zugmeyer et J.P. LE Cadre, "Méthodes d'intégration temporelle en traitement
d'antenne", Rapport Irisa $n^{\circ}$ 566, Rennes, décembre 90 .

[2] O. Zugmeyer et J.P. Le Cadre, "Méthodes paramétriques pour la détection de sources en mouvement", Rapport Irisa $n^{\circ} 567$, Rennes, décembre 90.

[3] G. Kitagawa and W. Gersch, "A smoothness priors time-varying $A R$ coeflicients of nonstationpriors time-varying AR cocflicients of nonstation-
ary covariance time series", IEEE Trans. on Aut. 